\title{
Archéopages
}

Archéopages

Archéologie et société

Hors-série 2 | 2010

Archéologie sans frontières

\section{La mission archéologique de Jebel Oust, site antique de Tunisie}

Raphaël Durost, John Scheid et Aïcha Ben Abed

\section{OpenEdition}

Édition électronique

URL : https://journals.openedition.org/archeopages/763

DOI : 10.4000/archeopages.763

ISSN : 2269-9872

\section{Éditeur}

INRAP - Institut national de recherches archéologiques préventives

\section{Édition imprimée}

Date de publication : 1 octobre 2010

Pagination : 46-51

ISSN : 1622-8545

\section{Référence électronique}

Raphaël Durost, John Scheid et Aïcha Ben Abed, «La mission archéologique de Jebel Oust, site antique de Tunisie ", Archéopages [En ligne], Hors-série 2 | 2010, mis en ligne le 01 octobre 2010 consulté le 23 février 2023. URL : http://journals.openedition.org/archeopages/763 ; DOI : https:// doi.org/10.4000/archeopages.763 


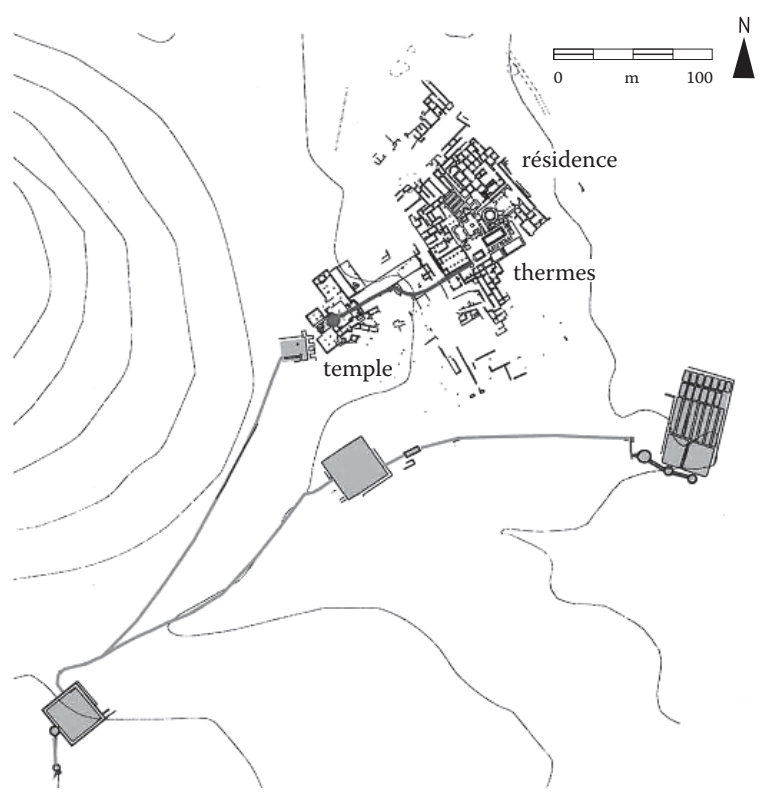

q

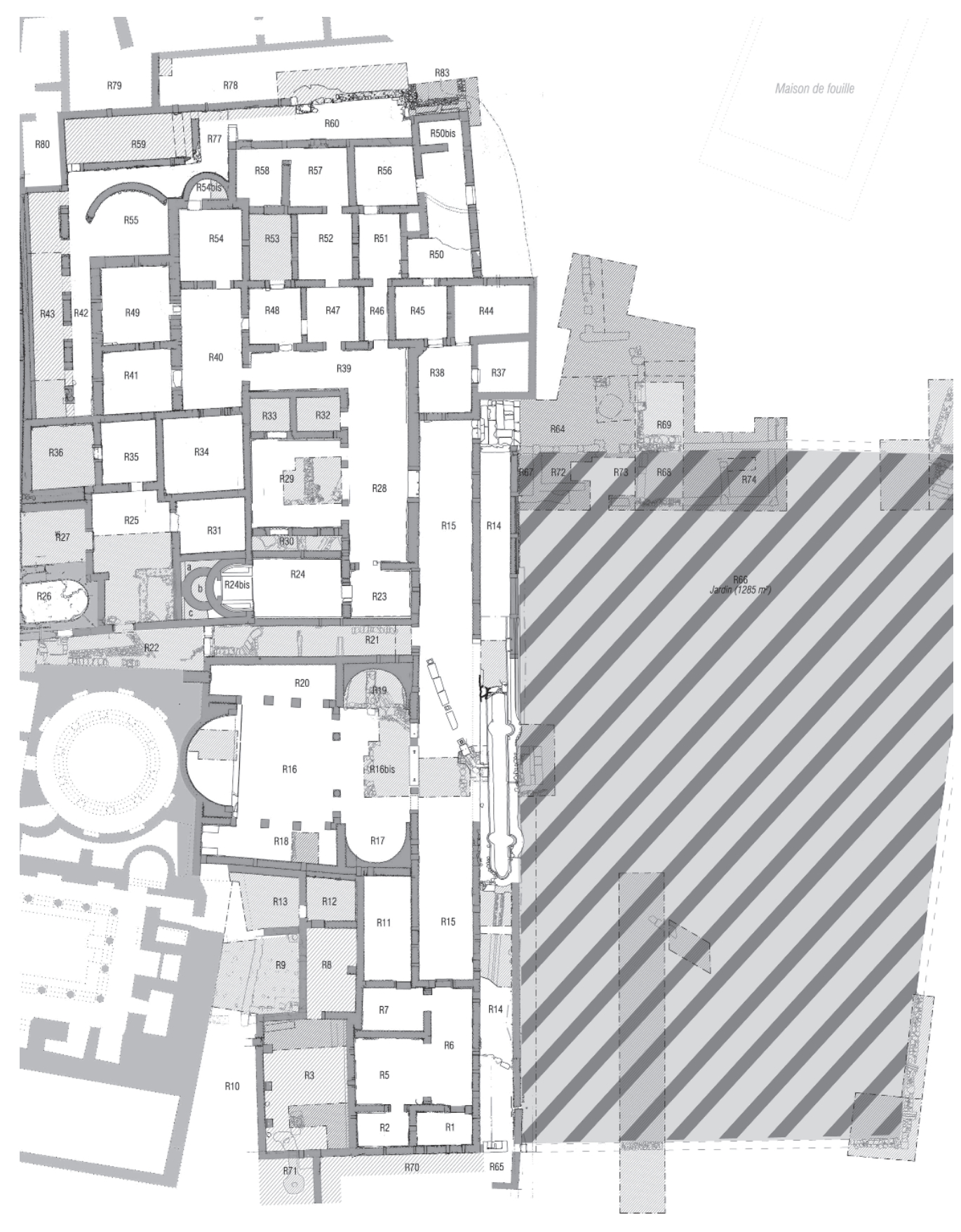

[Fig. 1] Plan général des constructions connues sur le site.

[Fig.2] Plan général de la résidence. De 2005 à 2010, le jardin

(zone hachurée) a fait l'objet de cinq sondages pour connaître ses contours et son contenu.

Les qualités agronomiques du sol sont probablement obtenues par des amendements réguliers. 


\section{La mission archéologique de Jebel Oust, site antique de Tunisie}

\author{
Raphaël Durost \\ Inrap \\ John Scheid \\ Collège de France \\ Aïcha Ben Abed \\ Institut national du patrimoine, Tunisie
}

$\mathbf{P}$ résentation générale du site. Le site antique appelé « Jebel Oust » est situé à

Hammam el-Oust, à $32 \mathrm{~km}$ au sud-ouest de Tunis, sur la route de Zaghouan. Il occupe la pente orientale du Jebel Oust, entre une source chaude (sur laquelle est construit un temple) et un secteur thermal équipé de deux portiques, donnant sur une résidence. Le temple, la source et les thermes ont été à l'origine du site, qui n'a sans doute jamais été une agglomération, mais plutôt un lieu de culte associé à une source thermale avec ses différentes annexes. Aucun matériel antérieur à l'Empire n'a été découvert à Jebel Oust, et l'histoire de cet ensemble s'étend entre le début de l'Empire et le début du VII ${ }^{\mathrm{e}}$ siècle. Après deux grandes étapes dans l'aménagement du site, vers la fin du $\mathrm{I}^{\mathrm{er}}$ siècle de notre ère et à partir du milieu du I $^{\mathrm{e}}$ siècle, avec deux implantations successives de thermes, l'une sur la pente devant le temple, l'autre au pied de la colline, les bâtiments sont christianisés au début du $\mathrm{v}^{\mathrm{e}}$ siècle. Le temple est transformé en baptistère et une basilique est construite en bordure du portique du temple. Le nom antique du site et l'identité de la divinité propriétaire du temple et de la source sont inconnus. L. Maurin a supposé qu'il pourrait s'agir du site Onellana, qui est mentionné sur la Table de Peutinger à égale distance de Oudhna et de Thuburbo maius (Ben Bassen, Maurin, 1998; Maurin, 2003), mais il n'y a aucune certitude. Il faut toutefois signaler que le temple de Jebel Oust est situé à proximité des limites de plusieurs cités ou pagi de vétérans. Étant donné sa probable naissance au début de l'Empire, il est raisonnable de l'attribuer à la colonie augustéenne d'Uthina (Oudhna) ou à l'un des deux pagi de vétérans augustéens situés entre Jebel Oust et Oudhna.

Historique des fouilles. Signalé pour la première fois en 1862 , le site n'a été l'objet de fouilles qu'en 1907. À l'occasion de la découverte, par un prospecteur minier, de la nappe phréatique qui sort à $55^{\circ} \mathrm{C}$, le bassin circulaire et la grande piscine rectangulaire dont les colonnes émergeaient encore de terre furent hâtivement fouillés par les militaires français (Carton, 1907). Le site fut ensuite oublié jusqu'en 1962, quand Mohammed Fendri dégagea les trois ensembles actuellement visibles, les thermes, le secteur du temple et les citernes. Une petite partie du matériel (non publié) a été déposée au musée d'Utique. Il a été rapatrié à Jebel Oust en 2003. Le reste des découvertes faites au cours de ces fouilles n'est pas conservé ou exploitable.

M. Fendri a fait dresser un plan de la zone de la résidence et des thermes, sur laquelle portait principalement la fouille de 1962-1963. Ce plan est d'excellente qualité et a conservé beaucoup de données, notamment sur l'aménagement des thermes, qui ont aujourd'hui disparu ou ont été abîmés au cours du temps. De la résidence, il livre peu de données de fouille. Le plan de la zone du temple, quant à elle, est conservé, mais dans un état très incomplet. Les citernes n'ont pas été relevées en détail. Enfin, ces différents plans n'étaient pas reliés entre eux. À part un article sur les mosaïques (Fendri, 1963) et deux conférences présentant le site et surtout les thermes de Jebel Oust, dont les manuscrits sont conservés, M. Fendri n'a laissé aucun document sur ses fouilles.

La mission actuelle de Jebel Oust a comme tâche la reprise du chantier de 1962, son relevé complet, létude des vestiges, au besoin par la fouille, et des couches encore en place, en vue de la publication du site. Cette équipe a été dirigée par Aïcha Ben Abed (INP) et John Scheid (Collège de France), avec la collaboration de Henri Broise (IrAa, CNRs), Catherine Balmelle (Centre Henri Stern, CNRs), Christophe Petit (Université de Bourgogne) et Jeannot Metzler (musée national d'Histoire et d'Archéologie de Luxembourg).

Objectifs de la mission archéologique : l'étude du jardin de la résidence. Le site de Jebel Oust se compose de trois unités : les thermes et les bâtiments qui les entourent, le temple et ses dépendances, ainsi qu'une série de grosses citernes, situées au sud, en marge du site proprement dit. Une fois le plan général établi, nous avons concentré les recherches sur le secteur du temple, sur les thermes et sur la résidence (Ben Abed, Scheid, 1905). C'est dans ce dernier secteur que la signature d'une convention avec l'Inrap a permis à un de ses archéologues d'intervenir de 2005 à 2010, ultime campagne de la mission. Cette étude s'est concentrée sur le jardin aménagé contre le côté de la résidence opposé aux thermes, au plus bas de la pente du Jebel Oust. La résidence est essentiellement connue par son dernier état, structuré à partir du IV ${ }^{\mathrm{e}}$ siècle et intégrant apparemment les thermes au cours du v $v^{e}$ siècle, et munie d'une salle de réception à absides ouvrant sur le jardin. Deux appartements donnant aussi sur le jardin jouxtent la salle de réception puis, sur le côté nord, une série de petites pièces carrées se succèdent. Leur fonction reste énigmatique car la plupart d'entre elles sont munies de deux ou trois portes donnant accès aux pièces suivantes, et l'espace disponible pour y séjourner se trouve donc fortement réduit.

L'intérêt archéologique du jardin est multiple. Tout d'abord, l'absence de maçonneries affleurantes a laissé penser à $M$. Fendri qu'il n'y avait pas de vestiges majeurs à cet endroit, et qu'il n'était pas prioritaire de l'explorer. Le jardin est donc parvenu au XXI ${ }^{e}$ siècle avec une stratigraphie intacte, chose rare sur le site. Il offrait la possibilité de documenter les phases tardives qui manquent ailleurs. Ensuite, la position en bas de pente a favorisé la préservation des strates successives, alors que les constructions des autres secteurs 

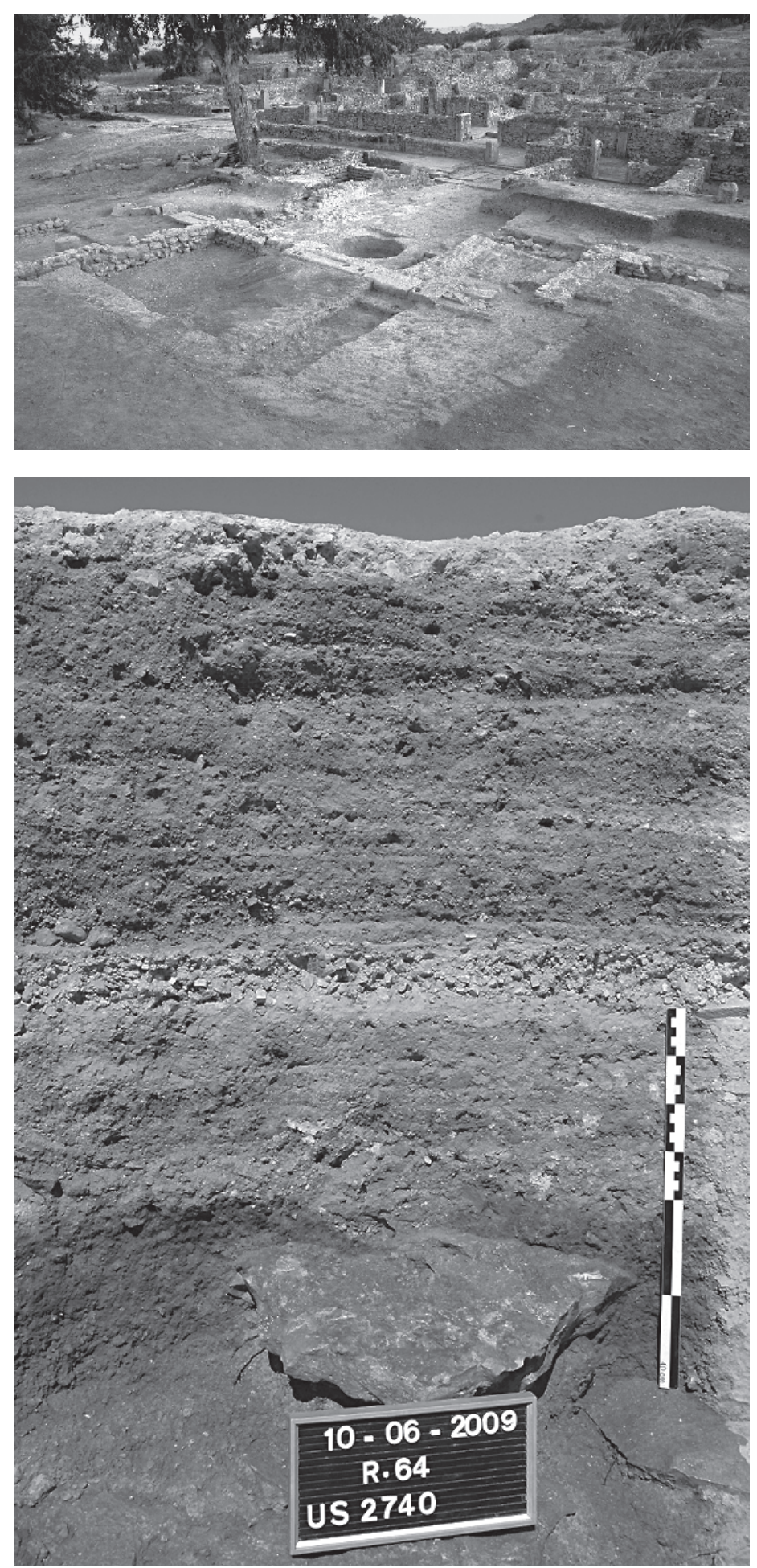

[Fig.3] Le sondage (angle ouest du jardin) a révélé des pièces sommaires

(au premier plan) dont l'étude permettrait de comprendre la destination du secteur résidentiel (habitat privé ou vocation publique et commerciale).
[Fig.4] L'angle ouest du jardin conserve un premier état à sol maçonné (non daté), recouvert de dépôts hydromorphes provenant d'au moins sept orages, démontrant une longue absence d'entretien avant que le sol de chaux de la résidence du IV ${ }^{\mathrm{e}}$ siècle ne soit installé. 
reposent, pour la plupart d'entre elles, directement sur la roche calcaire. Les phases les plus anciennes pouvaient donc être potentiellement préservées et il était envisageable de reconstituer les phases architecturales antérieures à la résidence du IV ${ }^{\mathrm{e}}$ siècle.

Les thèmes d'étude liés à un tel espace d'ornement végétal étaient extrêmement différents de ceux abordés dans le secteur cultuel, thermal et résidentiel. Il était nécessaire d'identifier des vestiges $a$ priori plus discrets, tels que des fosses de plantation ou des niveaux agricoles. Les compétences de l'Inrap ont été sollicitées pour cette raison, ses archéologues étant plus coutumiers de l'étude d'occupations sans maçonneries, avec des aménagements en creux. La densité moindre des aménagements attendus a également permis de mettre en œuvre certaines pratiques courantes en archéologie préventive : ouverture de sondages plus étendus que dans les autres secteurs, permettant de choisir les points d'échantillonnage de la stratigraphie, recours à un tracto pelle pour le décapage et prélèvement de sédiments pour des approches géomorphologiques (lames minces, palynologie, carpologie et malacologie).

L'exploration du jardin a tenu ses promesses. Les six campagnes qui lui ont été consacrées permettent de documenter l'histoire du site avant, pendant et après l'existence de la résidence telle qu'elle se présente à partir du $\mathrm{IV}^{\mathrm{e}}$ siècle. Durant ces deux siècles et le siècle suivant, le jardin forme une cour plane de $1285 \mathrm{~m}^{2}$ délimitée, sur son côté ouest, par les pièces de la résidence et, sur les trois autres côtés, par un simple mur. Le seul moyen connu d'y entrer se situe dans l'angle nord-ouest. Durant cette phase, un long et étroit bassin précède la vue du jardin depuis la grande salle de réception, et interdit de passer de l'un à l'autre. Ce n'est qu'au $\mathrm{V}^{\mathrm{e}} \mathrm{Ou} \mathrm{VI}^{\mathrm{e}}$ siècle que le point d'eau est comblé pour permettre de descendre à l'extérieur par trois marches et de rejoindre une allée.

Si l'intégralité du plan du jardin est loin d'être connue, les sondages démontrent qu'il s'agit d'un espace compartimenté en quartiers ornementaux et utilitaires. Les fosses de plantation identifiées au sein d'un sédiment aux qualités agronomiques extrêmement médiocres montrent que des espèces végétales sont plantées afin de composer un point de vue agréable depuis la salle de réception. Si la taille de certaines fosses peut accueillir des arbres, la découverte de pots horticoles dans les strates contemporaines du même secteur prouvent qu'il ne peut pas s'agir uniquement d'un verger. Notons par ailleurs que ces pots en céramique sont les premiers exemplaires découverts en Tunisie. Â l'instar de ceux connus dans la péninsule italique ou en Gaule, ils comportent trois trous ménagés à la base de la panse avant la cuisson. Contre le mur sud du jardin, à l'écart de la salle de réception, la nature du sol est différente. À la place d'espèces végétales plantées dans un substrat stérile, un niveau limoneux extrêmement organique est entretenu sur une trentaine de centimètres d'épaisseur. Son homogénéité suggère qu'il a fait régulièrement l'objet de labours, et les fréquents nodules de chaux rencontrés correspondent probablement à un apport volontaire destiné à atténuer l'acidité naturelle du terrain. Les tessons de céramique y abondent également, et appartiennent certainement aux déchets répandus comme fertilisant. Leur datation de la deuxième moitié du IV ${ }^{\mathrm{e}}$ jusqu'au VI $\mathrm{I}^{\mathrm{e}}$ siècle concorde avec la période d'occupation supposée du dernier état de la résidence, au cours duquel elle semble donc conserver son jardin sans modification majeure. L'abandon de ce dernier se situe à partir de la deuxième moitié du $\mathrm{vi}^{\mathrm{e}}$ siècle, terminus post quem du mobilier présent dans les niveaux qui marquent le recouvrement et l'arrêt de l'exploitation maraîchère du secteur. Deux prélèvements de cette couche organique ont été réalisés, l'un en colonne et le second en vrac, destiné à être tamisé pour étude carpologique et malacologique. Les premiers résultats tirés de la colonne stratigraphique montrent que le sol n'a pas conservé de pollens, et les autres études sont en cours.

En cherchant à localiser l'angle nord-est du mur du jardin, des constructions du $\mathrm{III}^{\mathrm{e}}$ siècle, antérieures à la résidence, sont apparues. Elles appartiennent à un bâtiment d'une largeur de $3 \mathrm{~m}$ et d'une longueur inconnue puisqu'il se poursuit de part et d'autre du sondage. Sa fonction tranche avec les espaces jusqu'à présent explorés sur le site, puisque il s'agit d'une zone de travail : un mur de refend divise l'espace étudié en deux pièces, dont l'une présente un aménagement singulier : six creux indurés sont alignés à intervalle régulier au-dessus d'un mur d'une cinquantaine de centimètres de haut. Ces creux, plus ou moins bien conservés, possèdent une induration plane sur le fond et, au mieux, sur trois côtés. Le quatrième côté, face au sud, est systématiquement ouvert. La rubéfaction environnante est très irrégulière, formant des contrastes aléatoires qui voisinent avec des endroits non rubéfiés. L'absence de rubéfaction du sol sous-jacent, à la base du mur, exclut l'hypothèse d'une sole suspendue de four, ou une suspensura d'hypocauste. Les plus fortes températures sont au contraire atteintes dans la partie supérieure. Ces creux sont plus probablement les empreintes de l'extrémité de six solives en bois servant à soutenir un plancher, leur autre extrémité reposant sur un mur situé hors du sondage. Le vide ménagé sous ce plancher correspondrait alors à un vide sanitaire, destiné à protéger de l'humidité du sol. Ce type de construction en bois paraît extrêmement rare, les comparaisons recherchées dans le cadre de cet article restant absentes en Afrique du Nord. La recherche d'un tel assainissement dans une seule pièce pourrait correspondre à un lieu de stockage de denrées alimentaires, telles que des céréales. Ces éléments démontrent qu'avant la construction de la résidence telle qu'elle se présente à partir du $\mathrm{IV}^{\mathrm{e}}$ siècle, un habitat existe dès le $\mathrm{III}^{\mathrm{e}}$ siècle, et qu'il possède au moins un secteur plus fonctionnel que résidentiel. Cet aspect inattendu rappelle que, dans un tel contexte rural, une résidence devait certainement tirer des ressources du terroir environnant, tout comme les établissements 

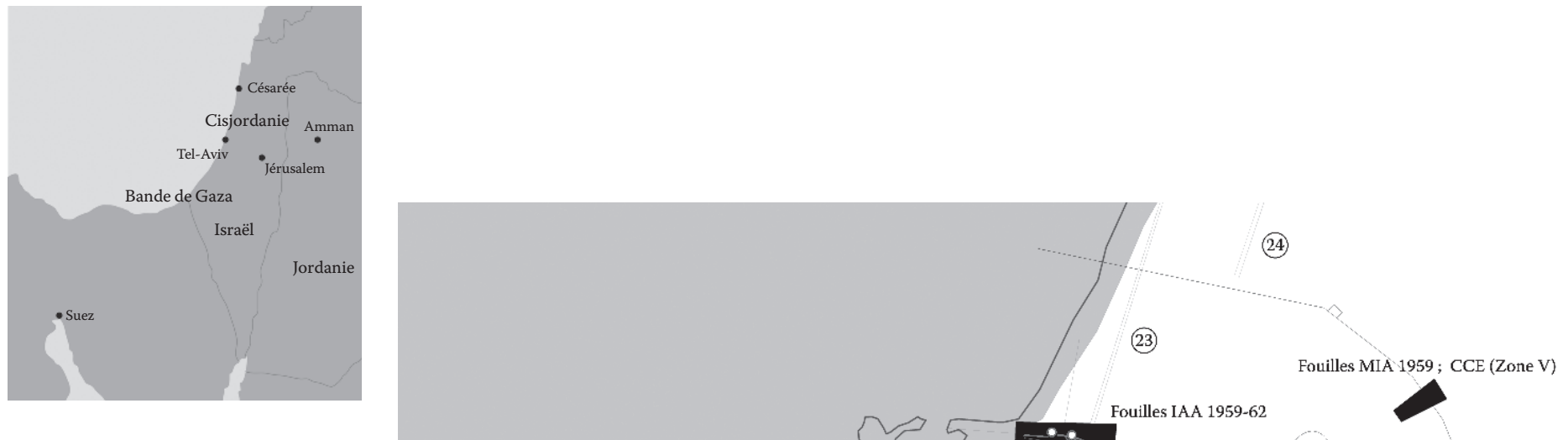

우

[Fig. 1] Carte d'Israël avec localisation de Césarée.

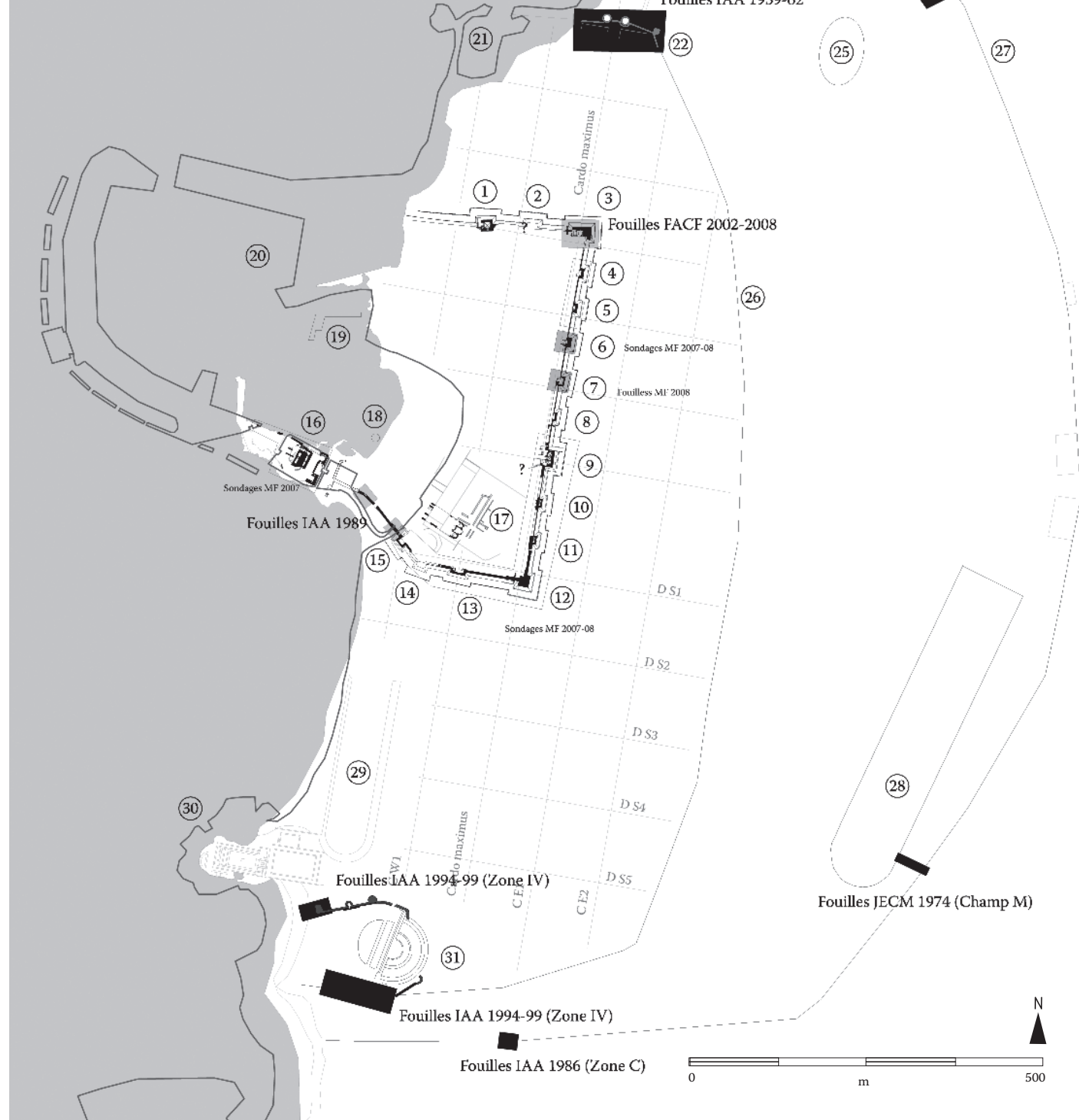

[Fig.2] Plan de l'historique des fouilles

1 - Tour 1 , porte nord

2 - Tour 2

3 - Tour 3

4 - Tour 4

5 - Tour 5

6 - Tour 6

7 - Tour 7

8 - Tour 8

9 - Tour 9, porte est

10 - Tour 10

11 - Tour 11

12 - Tour 12

13 - Tour 13

14 - Tour 14

15 - Tour 15 , porte sud-ouest
16 - Chateau

17 - Cathédrale

18 - Tour immergée

19 - Jetée des colonnes

20 - Avant-Port

21 - Port nord

tour polygonale

23 - Acqueduc

extérieur

24- Acqueduc

intérieur

25 - Amphitheâtre

26 - Enceinte

d'Hérode
22 - Porte nord et
27 - Enceinte

byzantine

28 - Hippodrome

29 - Cirque d'Hérode 30 - Palais d'Hérode

31 - Théâtre et son enceinte 
des campagnes romaines sur le continent européen. Quant au domaine reconstruit au cours du IV siècle, les moyens de subsistance de ses occupants restent inconnus, mais la suite de l'espace bâti au nord n'a jamais été explorée, et doit certainement contenir des réponses à ces questions.

En se consacrant au secteur dédié à l'ornementation végétale, ces campagnes ont apporté des informations qui intéressent la compréhension de la résidence de Jebel Oust. Les quelques résultats présentés ici montrent qu'en s'éloignant des aspects strictement architecturaux et en se penchant sur des secteurs marginaux, la compréhension des vestiges les plus spectaculaires du site s'améliore. L'exploitation de l'ensemble des données permettra de présenter un exemple richement documenté d'habitat rural de l'Afrique romaine.

\footnotetext{
BEN AbEd A., SCHEID J., 1905, « Nouvelles recherches archéologiques à Jebel Oust (Tunisie) », in CRAI, p. 321-349.

Ben BASSEn H., MAurin L. (ÉD.), 1998, Oudhna (Uthina). La Redécouverte d'une ville antique de Tunisie, Bordeaux, Paris, Tunis, p. 212.

Carton Dr., 1907, Bulletin de la Société Archéologique de Sousse, p. 22-23. CARTON DR., 1908, Comptes-rendus de l'Association française pour l'avancement des sciences, Congrès de Clermont-Ferrand.

CARTON Dr., 1914, Revue Tunisienne, p. 248.

FENDRI M., 1963, «Évolution chronologique et stylistique d'un ensemble de mosaïques dans une station thermale à Djebel Oust (Tunisie) », in La Mosä̈que gréco-romaine, colloque du CNRS, Paris, 1965, p. $157-173$.

Maurin L., 2003, Bir Mcherga 028 (S. Ben Baaziz, Carte Nationale des sites archéologiques et des monuments historiques), Tunis, p. 112
}

\section{Programme d'étude quadriennal des fortifications médiévales de Césarée}

\author{
Jocelyn Martineau \\ Inrap \\ Jean Mesqui \\ Société française d'archéologie \\ Nicolas Faucherre \\ Université de Nantes
}

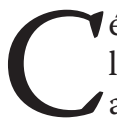

ésarée, Caesarea Maritima, est située sur la côte méditerranéenne d'Israël, à $60 \mathrm{~km}$ au nord de Tel-Aviv et à $40 \mathrm{~km}$ au sud d'Acre [Fig.1]. Devenue, depuis une vingtaine d'années, l'un des sites archéologiques les plus visités d'Israël, elle a été le siège d'une activité archéologique considérable à partir des années 1950, visant à révéler son passé antique et médiéval. Les fouilles menées entre les années 1960 à 2000 se sont concentrées

1 Professeur à l'institut d'archéologie, Université hébraïque de Jésuralem. au-delà du périmètre strictement hérodien; et enfin, à la période franque (entre 1251et 1252), la construction par Louis IX d'une enceinte quadrangulaire rétractée sur le port, seule subsistante de l'œuvre considérable de ce roi pour protéger les ports de la côte. La délimitation de la ville du Haut-Moyen Âge, après la conquête arabe au $\mathrm{VII}^{\mathrm{e}}$ siècle, restait à préciser, même si les fouilles menées à l'ouest du théâtre montraient un secteur urbain vivace à cette période. Le dégagement de la dernière enceinte, celle de saint Louis, dans les années 1960, a montré qu'elle constituait en fait une refortification d'une enceinte plus ancienne de même tracé. Elle fut d'abord attribuée aux Francs et datée de la période suivant leur conquête (1101). Mais plusieurs observations d'Israël Levine, et des découvertes archéologiques ponctuelles faites par Joseph Porath, au sud de l'enceinte franque, ont amené les archéologues israéliens à supposer que l'enceinte primitive existait déjà à l'époque islamique ancienne ; cependant, les indices étaient trop ténus pour la dater précisément comme pour la replacer dans son contexte urbain. La seule intervention archéologique programmée ensuite remonte à 2004 et portait sur un élément de courtine de la tour 3 .

La mission française de Césarée, menée entre 2007 et 2010, avait donc pour objectif général de préciser le plan et la chronologie de cette troisième enceinte essentiellement attribuée aux Francs. Le financement de l'opération a reposé sur la subvention du ministère français des Affaires étrangères et européennes, complétée par des subventions du Centre d'études supérieures de civilisation médiévale de l'Université de Poitiers (CESCM) et du Centre de recherche français de Jérusalem (CRFJ). Une convention de coopération, entre l'Institut national de recherches archéologiques préventives (Inrap) et la direction des Antiquités israéliennes (IAA), a par ailleurs permis de bénéficier de l'assistance de deux collègues de l'Inrap (un archéologue spécialiste du bâti médiéval et un topographe).

\section{Déroulement et résultats des campagnes.} Sur un linéaire d'un kilomètre de longueur, il était nécessaire d'adopter une approche pragmatique de l'enceinte médiévale de Césarée, une fouille systématique n'étant pas envisageable. Elle l'était d'autant moins que l'enceinte était archéologiquement très mal connue. Nous avons opté pour une approche double reposant, d'une part, sur le relevé systématique des structures en place au-dessus du sol (comprenant la topographie, le relevé architectural et l'analyse des maçonneries) et, d'autre part, sur la fouille exhaustive de deux tours de l'enceinte, les tours 6 et 7 . Le choix de ces deux ouvrages, effectué après la campagne 2007, ne tient ni au hasard, ni à l'opportunité; en effet, la tour 6 était la seule de l'enceinte à présenter de façon manifeste une élévation interne antérieure à Louis IX, alors que la tour 7 offrait une structure complexe qui autorisait à envisager le processus constructif. du temple et la frange littorale occidentale

d'un quartier byzantin et arabe ancien [Fig.2].

Bien que l'enceinte médiévale de la ville ait été reconnue depuis le $\mathrm{XIX}^{\mathrm{e}}$ siècle, ces fortifications nont pas été une priorité des recherches depuis leur dégagement sous la direction de Avraham Negev (entre 1959 et 1963). À ce moment, l'histoire des fortifications de Césarée se résumait à trois périodes d'aménagement : à la fin du i ${ }^{\mathrm{er}}$ siècle avant notre ère, la première ligne de défense, élevée sous Hérode, avec la fondation de la première ville; entre le $\mathrm{v}^{\mathrm{e}}$ et le $\mathrm{VI}^{\mathrm{e}}$ siècle, à la période byzantine tardive, la création d'une grande enceinte ovalaire 